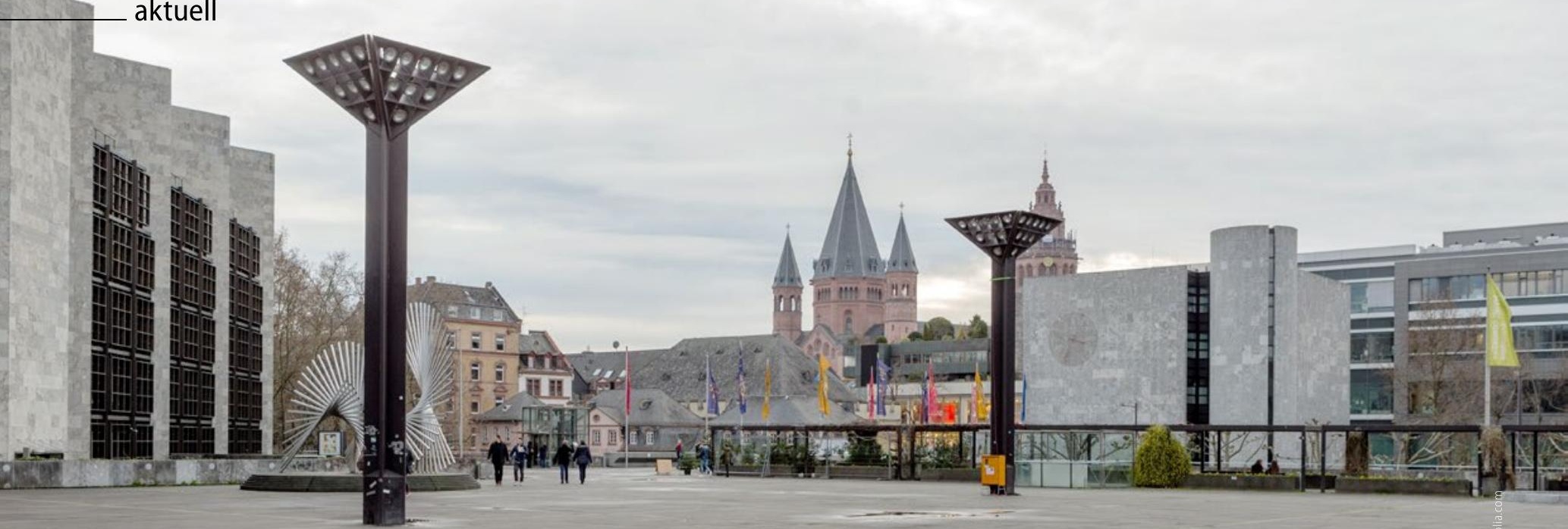

\title{
Topaktuelle Daten professionell aufbereitet und vermittelt
}

Bei der zweitägigen neurologischen Fortbildungsveranstaltung kamen in Mainz mehr als 500 Neurologen aus Klinik und Praxis auf ihre Kosten: Namhafte Referenten präsentierten zum achten Mal praxisrelevant und unterhaltsam die wichtigsten Forschungsergebnisse des zurückliegenden Jahres, diesmal mit 14 Themenschwerpunkten der Neurologie.

\section{Karpaltunnelsyndrom: Physiotherapie ist einen Versuch wert}

\section{Beim Karpaltunnelsyndrom wird gerne der Nerv operativ entlastet. Die Ergebnisse einer aktuellen Studie zeigen, dass man es auch erstmal nicht invasiv versuchen sollte.}

Die Entlastungsoperation ist beim Karpaltunnelsyndrom (KTS) wirksam und oft nicht vermeidbar, erklärte Professor Arne May, Institut für systemische Neurowissenschaften, Universitätsklinikum Hamburg-Eppendorf. Als „Highlight“ bezeichnete er eine Arbeit, in der eine physikalische Methode vorgestellt wurde, die dem Vergleich mit der Dekompensations-OP standhielt [Fernández-de-Las Peňas C et al. J Pain 2015; 16: 1087 -94]. In der Studie waren 120 Frauen mit KTS therapiert und ein Jahr nachverfolgt worden: 60 Patientinnen unterzogen sich einer Dekompressions-OP des Karpaltunnels und 60 weitere erhielten wöchentlich drei Physiotherapiesitzungen à 30 Minuten mit dem Ziel, den Nerv zu desensitivieren, erläuterte der Schmerzexperte. Als primäres Untersuchungsziel wurde die Schmerzintensität, als sekundäre Zielparameter die Funktion der Hand,
Subskalen des „Boston Carpal Tunnel Questionnaire“ und die Selbsteinschätzung der Patientinnen von einem verblindeten Untersucher zur Baseline sowie nach einem, drei, sechs und zwölf Monaten erfasst. 111 Patientinnen (92\%) beendeten die Studie nach zwölf Monaten, davon erhielten 55 eine physikalische Therapie und 56 wurden operiert (Intent-to-treat Analyse).

$\mathrm{Zu}$ den ersten beiden Untersuchungszeitpunkten war die Physiotherapie wirksamer als die Operation $(\mathrm{p}<0,01)$, später holte die OP aber auf, das heißt, beide Therapien führten in allen $\mathrm{Pa}-$ rametern zum gleich guten Erfolg, so May. „Die vergleichbaren Effekte sprechen dafür, die nicht invasive Methode vor der Entscheidung für eine Operation zumindest zu erwägen“, empfahl May. Kritisch merkte er an, dass die Publikation keinen Aufschluss darüber gibt, wie lange behandelt werden muss. Die erste Nachuntersuchung in der Studie nach vier Wochen lasse aber auf eine Behandlungsdauer von zwei bis vier Wochen schließen.

Ute Ayazpoor, freie Medizinjournalistin

Schmerz/Kopfschmerz (Arne May), 8. Neurologie-Update-Seminar, 19. - 20.2.2016 in Mainz 